\title{
Diffusiophoresis as ruling effect: Influence of organic salts on thermodiffusion of iron oxide nanoparticles
}

\author{
André Luiz Sehnem and Antônio Martins Figueiredo Neto \\ Institute of Physics, University of São Paulo, São Paulo CEP 05508-090, Brazil \\ Doreen Niether \\ ICS-3 Soft Condensed Matter, Forschungszentrum Jülich GmbH, D-52428 Jülich, Germany \\ Simone Wiegand \\ ICS-3 Soft Condensed Matter, Forschungszentrum Jülich GmbH, D-52428 Jülich, Germany \\ and Department für Chemie-Physikalische Chemie, Universität zu Köln, 50939 Cologne, Germany
}

(Received 4 October 2018; published 26 December 2018)

\begin{abstract}
Colloidal particles, including ferrofluidic nanoparticles (NP), move in a temperature gradient due to thermodiffusion. Organic salts, which are often added to disperse the NP in aqueous solution, also move in the temperature gradient. This can have a strong influence on the behavior of the NP, which not only respond to the temperature gradient but also to the concentration gradient of the dispersive salt, an effect termed diffusiophoresis. In this work we present experimental results on thermodiffusion of iron oxide nanoparticles dispersed in aqueous solutions of organic hydroxides, which illustrate the possibility to manipulate the thermodiffusion of NP through the addition of organic salts. Our experiments investigate the temperature dependence of the particles' Soret coefficient, a recurring question on thermodiffusion of water-dispersed particles. Existing theoretical approaches are compared and we relate the Soret coefficient of the NP with two main physical parameters ruling particle motion: the NP's electrostatic potential and the Soret coefficient of the dispersing ions. These parameters are also experimentally determined. At the order of magnitude of the NP's Soret coefficient good agreement between experiments and theory is achieved by including the experimental data on the Soret coefficient of the dispersing ions and therefore the NP's displacement due to the ion concentration gradient. Taking into account the temperature dependence of such previously unknown parameters is a relevant step to describe the temperature dependence of the NP's Soret coefficient.
\end{abstract}

DOI: 10.1103/PhysRevE.98.062615

\section{INTRODUCTION}

The presence of a temperature gradient induces the movement of solute molecules and particles dispersed in liquid solution, either to the cold or hot side [1-5]. This effect is named Soret effect, thermodiffusion, or thermophoresis [6-8]. It creates a steady concentration gradient of particles throughout the sample when the amplitude of thermodiffusive motion equalizes the counterflux induced by the concentration gradient. In this condition of stationary total flux of particles, the Soret coefficient $S_{T}$ is defined as $S_{T}=D_{T} / D$, where $D_{T}$ is the thermal diffusion coefficient and $D$ the Fickian diffusion coefficient. If the particles move to the cold side, then one defines the sign $S_{T}>0$ and to the hot side $S_{T}<0$.

The thermodiffusion effect has gained importance in the fields of nanofluidics and microfluidics in the context of microscale manipulation of dispersed micro- and nanoparticles [9-12]. Ferrofluid (iron oxide) nanoparticles have been proposed as nanocarrier agents for drugs and other molecules $[13,14]$, flowing through a liquid solution to reach a specific target. The intrinsic magnetic moment of the particles allows their manipulation by a magnetic field [15]. However, many works showed these particles also to have a strong thermodiffusive response when dispersed in water [16-18]. To effectively manipulate the movement of charged nanoparticles to the cold or hot side of a temperature gradient it is necessary to know the physical mechanisms underlying the thermodiffusive movement, some of which are as of yet insufficiently described by theory.

Theoretical models have been developed to predict values for the Soret coefficient of charged nanoparticles in magnetic colloids [19,20], but they predict the same influence for any type of dispersing ions, which disagrees with experimental observations. To elucidate the main mechanisms driving thermodiffusion of charged colloidal particles, including ferrofluids, some works performed systematic experimental investigation on the influence of specific parameters, like particle size [21-23], type of dispersive salt [24-26], and particle-solvent interface $[27,28]$. The Soret coefficient of charged particles in general has been described as having many physical contributions: thermoelectric fields generated by charged species in solution $[17,23,25,26,29]$, temperature dependence of the electrostatic energy in the electric-double layer [3,30-33], motion induced by ionic concentration gradients $[34,35]$, and solution thermal expansion [36,37], as examples. It means that the experimentally obtained Soret coefficient can be described either as a sum of all the mechanisms taking place [16] or simply by the stronger ones [38]. It is possible to observe one 
kind of mechanism by controlling the physical parameters of the solution, like salt [29] or surfactant concentration [28]. Solute molecules and charged particles in water typically show a temperature dependence of the Soret coefficient described by an empirical equation, suggested by Iacopini et al. [39],

$$
S_{T}(T)=S_{T}^{\infty}\left(1-e^{\left(T^{*}-T\right) / T_{0}}\right)
$$

where $S_{T}^{\infty}, T^{*}, T_{0}$ are empirical fitting parameters not related to any theoretical approach. The origin of such behavior as being due to one or more of the above-mentioned physical mechanisms is not clear in literature. Some works suggest that the empirical thermodiffusion behavior could be caused by the temperature dependence of the hydrogen bond network in water $[40,41]$.

This work investigates experimentally the Soret coefficient of magnetite $\left(\mathrm{Fe}_{3} \mathrm{O}_{4}\right)$ nanoparticles dispersed in aqueous solutions of organic hydroxides, TMAOH (tetramethylammonium hydroxide) and TBAOH (tetrabutylammonium hydroxide). The Soret coefficient is determined by infrared thermal diffusion forced Rayleigh scattering (IR-TDFRS). While the experimentally observed IR-TDFRS signal due to the Soret effect is dominated by diffusion of the nanoparticles, it is strongly influenced by the Soret response of the dispersive ions. The temperature dependence for $S_{T}$ does not always follow Iacopini equation as the hydroxide concentration increases. The analysis based on the up-to-date theoretical models shows a clear relation between the Soret coefficient of ions, measured in solution without particles, and the NP's Soret coefficient when dispersed in the ionic solutions. In the following section we present the experimental procedure to obtain the Soret coefficient of ions and NP by the holographic experiments in IR-TDFRS. After that we compare the measured values of the NP's Soret coefficient with theoretical predictions. We show that the experimental data to the temperature dependence of the NP's Soret coefficient is proportional to the Soret coefficient of the added electrolytes. The influence of an internally generated diffusiophoretic effect will be discussed. An equation based on this effect is introduced, which describes correctly the observed tendency in experimental results.

\section{EXPERIMENTAL SECTION}

\section{A. Sample}

The ferrofluid solution investigated in this work consists of iron oxide nanoparticles of magnetite $\mathrm{Fe}_{3} \mathrm{O}_{4}$ dispersed in water and stabilized by $\mathrm{NaOH}$ (sodium hydroxide). The nanoparticles were purchased from Chemicell $\mathrm{GmbH}$, namely, the ferrofluid FluidMAG-UC/A. They were synthesized by the coprecipitation method and the hydrodynamic diameter given as $d_{h}=50 \mathrm{~nm}$. The particles have been characterized previously by XRD, DLS, and SAXS (see Supplemental Material [42] for details), and it was found that the diameter is overestimated due to the contribution of larger particles or aggregates $(\approx 2 \%)$. For evaluation of Eqs. (7) and (8) we used $d_{h}=35$ $\mathrm{nm}$, which was calculated from the diffusion coefficient measured with IR-TDFRS by using the Stokes-Einstein equation. This value agrees well with the previous findings. The original solution has a volumetric particle concentration of $25 \mathrm{mg} / \mathrm{ml}$ and a low concentration of free hydroxides $(\mathrm{pH} \approx 8)$. This initial low ionic concentration will be neglected when the organic salts are added, since it is orders of magnitude smaller. The addition of organic hydroxides does not change the colloidal equilibrium in the timescale of the experiments and its purpose is the investigation of the ionic influence on the NP's Soret coefficient. The thermodiffusion experiments were performed in samples with particle concentration of $5 \mathrm{mg} / \mathrm{ml}$, by dilution of the original solution with pure water and the desired amount of hydroxides.

The organic hydroxides tetramethylammonium hydroxide (TMAOH) and tetrabutylammonium hydroxide (TBAOH) were added to the diluted nanoparticle solution to achieve the desired ionic concentrations. The aqueous solutions of TMAOH and TBAOH were purchased from Sigma Aldrich and used without further purification.

\section{B. Experimental setups}

\section{Zeta potential and electric conductivity determination}

The nanoparticles' $\zeta$ potential was measured employing the well-known electrophoretic light scattering method. We used a commercial equipment (Brookhaven ZetaSizer 90 Plus, with electrodes for polar solvents, model AQ-1154). The $\zeta$ potential determination in our samples was limited to ionic concentrations below $1 \mathrm{mMol} / 1$ of added hydroxides, as the signal stability is low for higher ionic concentrations. The electric conductivity is measured simultaneously. Temperature control is done internally by the equipment with precision $\Delta T=0.1 \mathrm{~K}$.

Experiments were done with the diluted original solution and solutions where nanoparticles are dispersed in TMAOH and $\mathrm{TBAOH}$, with particles concentration of $5 \mathrm{mg} / \mathrm{ml}$ in all solutions. The original solution is stabilized by a very low concentration of $\mathrm{NaOH}(c<0.01 \mathrm{mMol} / \mathrm{l})$ and concentrations of $0.5 \mathrm{mMol} / 1$ and $1 \mathrm{mMol} / 1$ of added organic hydroxides were measured. The electric conductivity was also measured in aqueous solutions of hydroxides containing the same amount of ions added to the nanoparticles solutions.

\section{Soret coefficient measurement}

We used IR-TDFRS to obtain the nanoparticles' Soret coefficient and diffusion coefficient. Details about this setup are described elsewhere [43]. The temperature grating is induced with an infrared laser, which is absorbed by water [44]. The experiment is frequently used for thermodiffusion experiments in binary liquid mixtures and liquid solutions of ions, molecules and nanoparticles.

The working principle of the technique is the following: an infrared laser (wavelength $\lambda_{w}=980 \mathrm{~nm}$, with a beam power $P \approx 0.5 \mathrm{~W}$ ) is split into two beams that are directed by two mirrors to the same spot inside the sample. The interference of the two beams generates a sinusoidal periodic intensity grating. Light absorption by the sample induces a periodic temperature profile and the Soret effect moves particles in solution either to the high or low temperature. The induced gratings of temperature and concentration create a refractive index grating that diffracts a read-out beam of wavelength $\lambda_{r}=632.8 \mathrm{~nm}$ and beam power $P \approx 30 \mathrm{~mW}$. The diffracted light from the read-out beam is recorded. The build up of the concentration grating due to the Soret effect is several orders 
of magnitude slower compared to the temperature grating, as the value of thermal diffusivity $D_{\text {th }}$ is much higher than the particle's mass diffusion $D$. At least 1000 independent measurements were performed in each experiment.

\section{Soret coefficient evaluation}

For a binary system the amplitude of the total heterodyne scattered signal $\zeta_{\text {het }}(t)$ is normalized by the thermal grating signal and its final amplitude is related to the Soret coefficient by the expression:

$$
\begin{aligned}
\zeta_{\text {het }}(t)= & 1-\exp \left(-\frac{t}{\tau_{\text {th }}}\right)-\frac{A}{\tau-\tau_{\text {th }}}\left\{\tau\left[1-\exp \left(-\frac{t}{\tau}\right)\right]\right. \\
& \left.-\tau_{\text {th }}\left[1-\exp \left(-\frac{t}{\tau_{\text {th }}}\right)\right]\right\},
\end{aligned}
$$

where $\tau_{\text {th }}$ is the heat diffusion time defined as $1 /\left(q^{2} D_{\text {th }}\right)$, $\tau$ is the time constant related to the translational collective diffusion coefficient as $1 /\left(q^{2} D\right), q$ is the modulus of the scattering vector that can be obtained from the measured fringe spacing $d, d=2 \pi / q$. The amplitude $A$ is given by

$$
A=\frac{(\partial n / \partial \phi)_{p, T}}{(\partial n / \partial T)_{p, \phi}} S_{T} \phi(1-\phi),
$$

where $\phi$ is the particles volumetric fraction in solution. For a ternary mixture [45],

$$
\zeta_{\text {het }}=1-e^{-t / \tau_{\mathrm{th}}}-A^{\text {salt }}\left(1-e^{-q^{2} D_{\text {salt }} t}\right)-A^{\mathrm{NP}}\left(1-e^{-q^{2} D_{\mathrm{NP}} t}\right),
$$

where $D_{\text {salt }}$ and $D_{\mathrm{NP}}$ are the diagonal elements of the matrix of diffusion coefficients. In analogy to binary mixtures, cf. Eq. (3), we relate the amplitudes to the Soret coefficients, $S_{T}$, for each component from the ratio of mass fraction and temperature gradients in the steady state, where the mass flows vanish:

$$
\begin{aligned}
A^{\text {salt }} & =\frac{(\partial n / \partial w)_{P, T, \phi}}{(\partial n / \partial T)_{P, w, \phi}} w(1-w) S_{T}^{\text {ion }}, \\
A^{\mathrm{NP}} & =\frac{(\partial n / \partial \phi)_{P, T, w}}{(\partial n / \partial T)_{P, w, \phi}} \phi(1-\phi) S_{T}^{\mathrm{NP}} .
\end{aligned}
$$

Only recently, ternary systems have been investigated in more detail [46]. Especially the investigation of ternary liquid mixtures can become quite tedious due to the strong coupling of the cross diffusion coefficients. The analysis becomes easier when a polymer or colloidal particle is dispersed in solution at very low volume concentrations [45]. Under these circumstances it is often possible to separate the mass diffusion of the dispersed particle from the movement of other molecules due to the fact that the diffusion coefficients differ by more than an order of magnitude. In that particular case it was possible to derive the binary Soret coefficient of the solvent mixture from the analysis of the ternary system.

In principle, we have a quite similar situation dispersing nano particles in an aqueous solution of an organic hydroxide. The main difference to the previous study is that we deal with charged systems, which might cause different effects. For instance it might be more difficult to observe an independent movement of the organic salt due to adsorption of the ions on the surface of the colloidal nano particle.

For the concentrations of organic salts used in this study a concentration signal due to the ionic gradient, quantified by $A^{\text {salt }}$, could not be determined. Fixing the equilibration time to the one observed for the binary mixture (organic salts in water) and using Eq. (4) the Soret coefficient of the NPs changed by up to $4 \%$ when $20 \mathrm{mM}$ of hydroxide is added and only up to $1 \%$ when $2 \mathrm{mM}$ or less of hydroxide is added to the solution. Unfortunately, it is not possible to quantify the Soret coefficient of the small ions from the measurement of the ternary system as in the work of Kita et al. [45], but we find a clear evidence for the movement of organic salt that justifies the diffusiophoretic contributions to the Soret coefficient of the NPs.

Due to the small contribution of the organic salts to the concentration signal, the evaluation of the nanoparticles Soret coefficient is made by considering the system as a binary solution. This means that thermal diffusion signal $\zeta_{\text {het }}(t)$ is dependent only on the NP's thermodiffusion. The best fit of Eq. (2) to the experimental data $\zeta_{\text {het }}(t)$ gives the values for $D_{\text {th }}, D$, and $S_{\mathrm{T}}$. The particles' thermal diffusion coefficient is calculated by $D_{\mathrm{T}}=D S_{\mathrm{T}}$. For the complete investigation of thermodiffusion in ferrofluids we did experiments in nanoparticle solutions containing hydroxides and separately in the aqueous solutions of hydroxides. The results to the Soret effect of hydroxides in water are discussed elsewhere [47], and are used here to describe the Soret coefficient obtained for ferrofluid nanoparticles. We use the previous results of low concentration dependence for the Soret coefficient of the hydroxides [47] to perform the theoretical analysis and evaluate the particle's Soret coefficient at lower hydroxide concentration.

In Fig. 1 we illustrate the physical mechanisms occurring in the holographic experiments when measuring the particles' Soret coefficient. The temperature gradient induces the Soret effect in ions and nanoparticles. The experiments take the time necessary for the flux of particles to reach a stationary state, reflected in the stationary light-intensity signal diffracted by the particles' concentration grating.

\section{RESULTS}

\section{A. Ionic Soret coefficient}

The Soret coefficient of TMAOH and TBAOH in water has been measured earlier and the effects of the ion's nature on ionic thermodiffusion were discussed [47]. Here we only present experimental results for $S_{T}^{\text {ion }}(T)$, which are at the low limit of concentration for possible measurements by the IR-TDFRS experiments. Figure 2 shows the temperature dependence of the aqueous solutions of TMAOH at $24 \mathrm{mMol} / 1$ and $\mathrm{TBAOH}$ at $20 \mathrm{mMol} / \mathrm{l}$.

\section{B. Zeta potential and electric conductivity}

The values obtained for the $\zeta$ potential at four different temperatures, spanning the range of temperatures of the thermodiffusion experiments, are presented in Table I. As the concentration of the hydroxides increases, the $\zeta$-potential values decrease in absolute value, indicating screening of the surface 

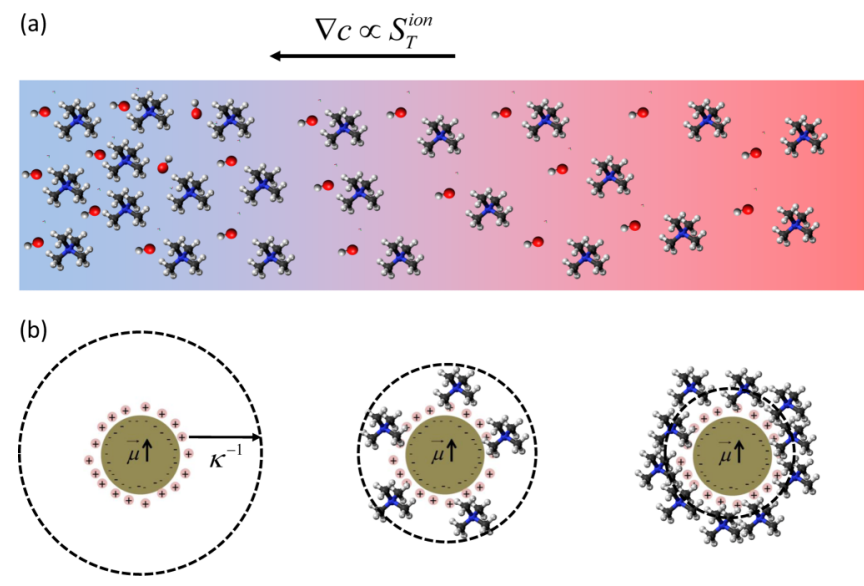

FIG. 1. (a) Illustration of Soret effect of the organic hydroxides in aqueous solutions. The image represents a half space period of the grating temperature, from a minimum (blue side) to a maximum (red side) in temperature. In a previous work [47] the Soret coefficient of ions $S_{T}^{\text {ion }}$ was experimentally determined. It was shown that the organic salts accumulate on the cold side of the temperature gradient. (b) Sketch (out of scale) of the addition of organic salt to the nanoparticle solution: an initially small amount of $\mathrm{NaOH}$ in the original solution induces a long-range electric field (large Debye length). The increasing concentration of the organic salts screens the particle's electric field.

charges. This corroborates the microscopic picture sketched in Fig. 1, where organic cations attach to the negatively charged surface of the NP. Such an attachment would lead to the observed lowering in $\zeta$-potential values but also be likely to induce changes in the NP's thermophoresis. The magnitude of the $\zeta$ potential decreases by $10 \%$ with increasing temperature at low ion concentration (cf. Table I). A similar observation has been made by Al Mahrouqui [48] in natural carbonates. They found that the magnitude of the $\zeta$ potential decreases with increasing concentration of the potential determining ion and proposed that the observed temperature dependence at low ionic strength is a buffering effect caused by the temperature dependence of $\mathrm{pH}$.

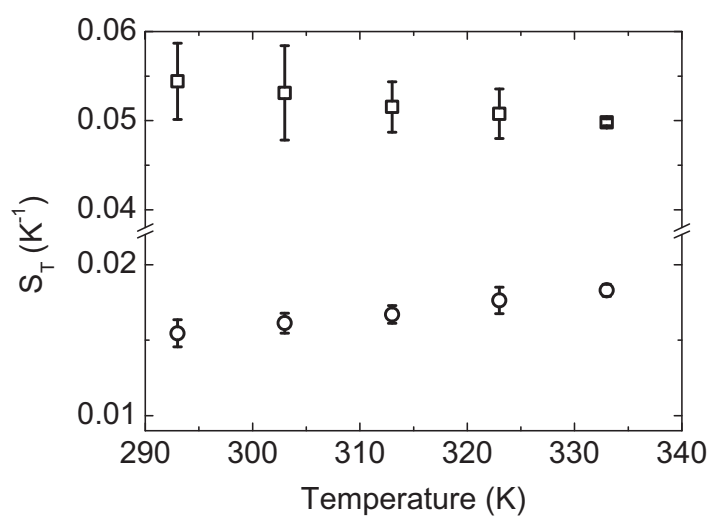

FIG. 2. Temperature dependence of the Soret coefficient of aqueous solutions of $24 \mathrm{mMol} / 1$ of TMAOH (open circles) and 20 $\mathrm{mMol} / 1$ of TBAOH (open squares) in water.
TABLE I. Zeta-potential values in volts obtained for the nanoparticles in different electrolyte concentrations as function of temperature.

\begin{tabular}{lccccc}
\hline \hline $\mathrm{T}$ & 0 & $0.5 \mathrm{mM}$ & $1 \mathrm{mM}$ & $0.5 \mathrm{mM}$ & $1 \mathrm{mM}$ \\
$(\mathrm{K})$ & $\mathrm{mM}$ & TMAOH & TMAOH & TBAOH & TBAOH \\
\hline 298 & -0.075 & -0.048 & -0.045 & -0.055 & -0.042 \\
308 & -0.07 & -0.044 & -0.041 & -0.047 & -0.039 \\
318 & -0.067 & -0.042 & -0.039 & -0.044 & -0.037 \\
328 & -0.062 & -0.039 & -0.037 & -0.04 & -0.037 \\
\hline \hline
\end{tabular}

It is known that, in the case of iron oxide nanoparticles, an increasing concentration of hydroxides leads to increasing absolute values of the particle surface charge $\sigma^{s}$, from $\sigma^{s} \leqslant$ $-0.05 \mathrm{C} / \mathrm{m}^{2}$ for $c^{\text {hydroxide }} \leqslant 0.01 \mathrm{mMol} / 1$ to $\sigma^{s} \sim-0.2 \mathrm{C} / \mathrm{m}^{2}$ for $c^{\text {hydroxide }} \sim 1 \mathrm{mMol} / 1[21,49]$, indicating a modification of the surface by the added salts. The equilibrium between particle surface and solution is represented by protonation and deprotonation reaction equations [21,50]. An increase in absolute surface charge values due to increasing occupation of surface charge sites by hydroxide ions also implies more counterions near to particles's surface, decreasing $\zeta$ potential values. We measured the electric conductivity for some concentrations of the organic hydroxides, as presented in Figs. 3(a) and 3(b). We observe a decrease of the electric conductivity of the salt solution when the nanoparticles are added. This indicates a reduction of ion mobility and shows that the salt ions added to the ferrofluid solutions reside to some extent in the particles' electric double layer, and the concentration of free ions in solution is lower than the added amount of hydroxides. This effect contributes to the influence the organic salts are observed to have on the nanoparticle's thermophoresis, which is presented in the next section.

\section{Thermodiffusion of the nanoparticles}

Figures 4(a) and 4(b) show the experimental results on the Soret coefficient of nanoparticles as function of temperature for five different concentrations of TMAOH and TBAOH. The concentration $c=0 \mathrm{mMol} / \mathrm{l}$ refers to the nanoparticle suspension without added salt, apart from the original small amount of $\mathrm{NaOH}\left(c<10^{-5} \mathrm{Mol} / \mathrm{l}\right)$. Solid lines are best fits with Iacopini's equation to the experimental results. The qualitative behavior of the results is reasonably well described by Iacopini's equation as the concentration of TMAOH increases, but the parameters obtained from this fit procedure have no physical meaning, apart from the inversion temperature $T^{*}$, which is clearly decreasing. When the concentration of TBAOH increases to $c \geqslant 0.5 \mathrm{mMol} / \mathrm{l}$, Iacopini's equation does not fit the experimenal results. Instead, $S_{T}(T)$ decreases with increasing temperature, a behavior explained by the results of the coefficients $D_{T}$ and $D$. The temperature dependence of $D_{T}(T)$ is stronger for the higher concentration of TBAOH compared to TMAOH [see Figs. 5(a) and 5(b)], while $D(T)$ changes with increasing salt concentration at the same rate for both salts. The consequence is the change in the slope of $S_{T}(T)$. In other words, a threshold of $D_{T}(T)$ values determines the positive or negative slope for $S_{T}(T)$ in our samples. 


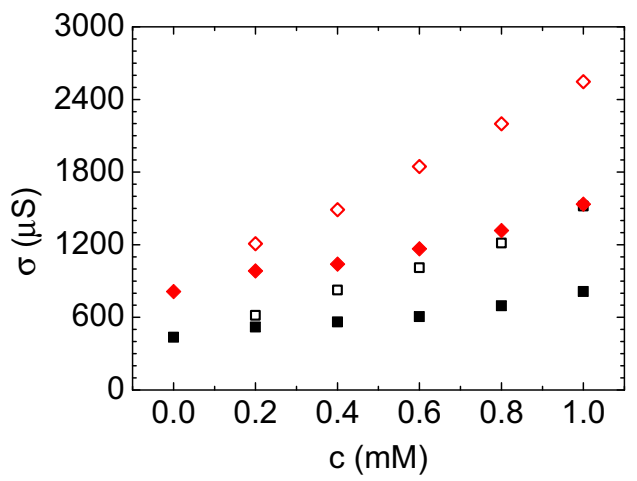

(a)

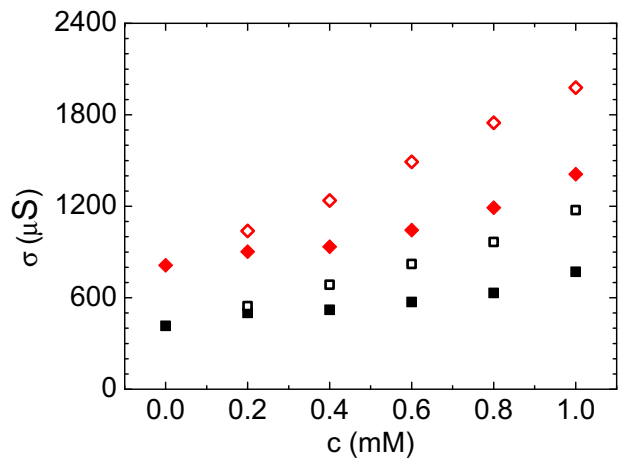

(b)

FIG. 3. Electric conductivity as function of salt concentration for aqueous solutions of (a) TMAOH and (b) TBAOH. Open symbols are the summed values for salts and nanoparticles when measured separately $\left(\sigma=\sigma^{\text {salt }}+\sigma^{\mathrm{NPs}}\right)$ and closed symbols are values for solutions of salts + nanoparticles $\left(\sigma^{\text {salt }+\mathrm{NPs}}\right)$, at $T=25^{\circ} \mathrm{C}$ (black squares) and $T=55^{\circ} \mathrm{C}$ (red diamonds).

Figures 5(a) and 5(b) show results of the thermal diffusion coefficient $D_{T}(T)$ of the nanoparticles at different concentrations of salts and of the aqueous salt solutions without nanoparticles. The highest $D_{T}$ values of the particles, corresponding to those obtained for $S_{T}(T)$, are seen for salt concentrations around $1 \mathrm{mMol} / \mathrm{l}$.

In the Discussion section we present a description on how the ionic concentration gradient influences the Soret effect of the nanoparticles. The results in Figs. 4(a) and 4(b) show that the $S_{T}$ values are shifted towards higher values for increasing salt concentrations and approach nearly constant values around $1 \mathrm{mM}$. It is remarkable that the Soret coefficient of the nanoparticles responds so sensitively to even minimal addition of the organic salts. We will discuss the physical effects which influence the nanoparticles Soret coefficient when salt is added to the solutions in the following section.

\section{DISCUSSION}

Based on the results presented in Figs. 4(a) and 4(b) and in previous works $[16,23,29]$, it is not only the concentration, but also the type of ions added to the solution that determines sign and amplitude of the nanoparticles' Soret coefficient $S_{T}^{\mathrm{NP}}(T)$.
Although it is several orders of magnitude larger, there is a suggestive relation of the temperature dependence between $S_{T}^{\mathrm{NP}}(T)$ and $S_{T}^{\text {ion }}(T)$ : the NP's Soret coefficient values (cf. Fig. 4) seem to reflect the temperature dependence obtained for the respective dispersing ions (cf. Fig. 2). On comparing the experimental results with theoretical predictions, we aim to explain the sign and order of magnitude of the nanoparticles when TMAOH and TBAOH are added to the nanoparticles solution.

A first attempt to describe our data by a theoretical relation between $S_{T}^{\mathrm{NP}}(T)$ and ionic Soret coefficient is by the use of the equation proposed by Eslahian et al. [23]. The equation for $S_{\mathrm{T}}^{\mathrm{NP}}(T)$ is

$$
S_{T}^{\mathrm{See}}=\frac{\varepsilon}{\eta T D}\left(\frac{\zeta^{2}}{12}(1+\tau+\alpha)-\zeta S T\right),
$$

where $\varepsilon, \eta$, and $\zeta$ are the dielectric permittivity, the viscosity, and the particle's electrokinetic potential, respectively. To estimate $S_{T}^{\text {See }}$ we use the dielectric permittivity and viscosity values for water, a good approximation for solutions with low concentrations of ions and particles [16]. The Seebeck coefficient is $S=\left(Q_{+}^{*}-Q_{-}^{*}\right) / 2 T e$, with $Q_{i}^{*}$ the ionic heat of

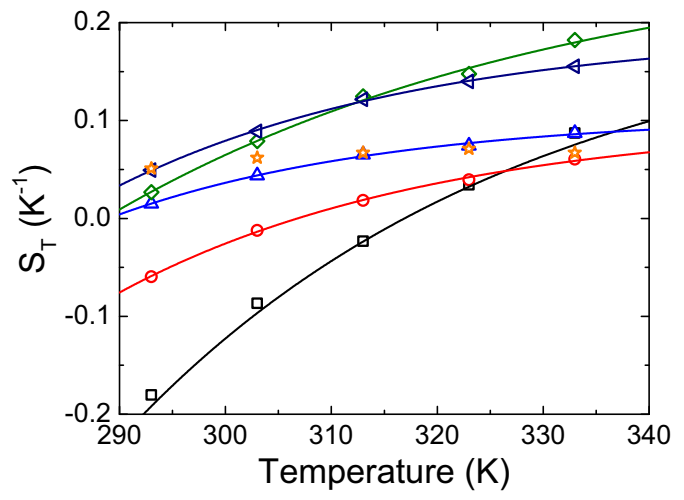

(a)

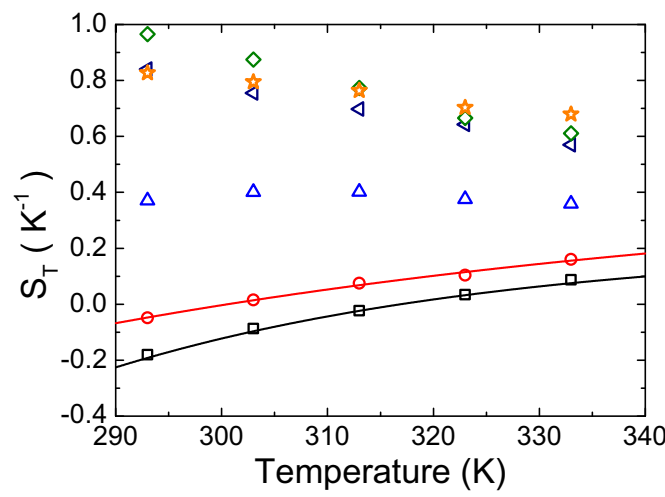

(b)

FIG. 4. Soret coefficient of the nanoparticles (particles concentration of $0.5 \mathrm{mg} / \mathrm{ml}$ ) as function of temperature for some concentrations of the added salts TMAOH (a) and TBAOH (b): $0 \mathrm{mMol} / 1$ (black squares), $0.1 \mathrm{mMol} / 1$ (red circles), $0.5 \mathrm{mMol} / 1$ (blue triangles), $1 \mathrm{mMol} / 1$ (olive diamonds), $2 \mathrm{mMol} / 1$ (navy triangles), and $20 \mathrm{mMol} / 1$ (orange stars). The solid lines in the corresponding colors are the best fit curves with Iacopini's equation $S_{T}(T)=S_{T}^{\infty}\left(1-e^{\left(T^{*}-T\right) / T_{0}}\right)$. 


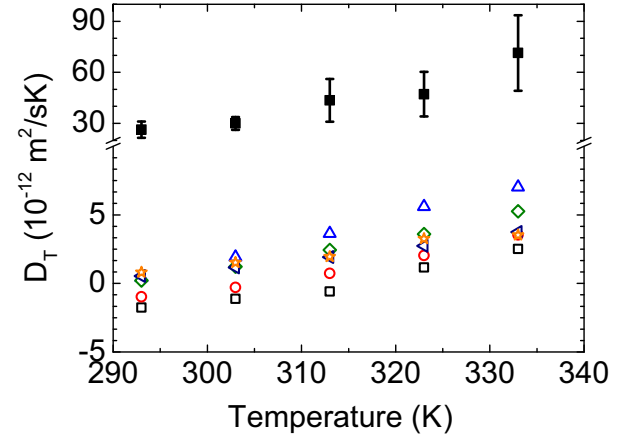

(a)

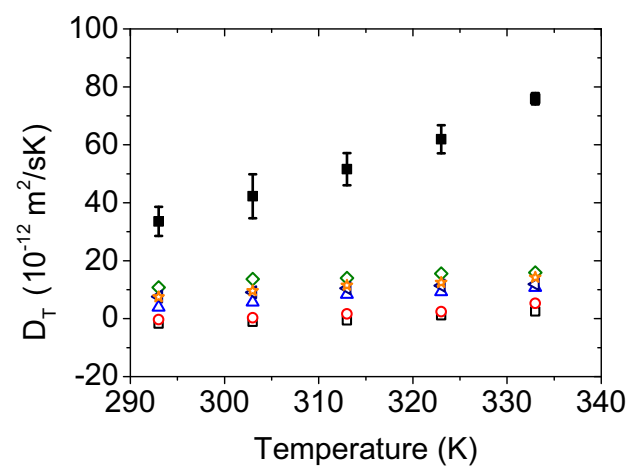

(b)

FIG. 5. Thermal diffusion coefficient $D_{T}$ as function of temperature for different concentrations of (a) TMAOH and (b) TBAOH. The hollow colored symbols indicate nanoparticles with the same concentrations of additive salt as in Figs. 4(a) and 4(b). The filled squares represent the values obtained for the aqueous solutions of salts $(24 \mathrm{mMol} / 1$ for TMAOH and $20 \mathrm{mMol} / 1 \mathrm{for}$ TBAOH $)$, also measured by the same experimental technique.

transport of cations and anions [51,52]. $Q_{i}^{*}$ was obtained by Agar et al. [53] at $T=298 \mathrm{~K}$ and ionic concentrations of 10 $\mathrm{mMol} / \mathrm{l}$; temperature and concentration dependence are not known. In Eq. (7) $\alpha=\left(Q_{+}^{*}+Q_{-}^{*}\right) / 2 k_{b} T$ is defined as the ionic Soret coefficient, which can alternatively be described by the experimental values with $\alpha=T S_{T}^{\text {ion }}$. The temperature dependent $S_{T}^{\text {ion }}$ values of TBAOH and TMAOH are taken from Fig. 2 for $20 \mathrm{mM}$ and from earlier measurements [47], extrapolated to infinite dilution for $1 \mathrm{mM}$. The diffusion coefficient of the nanoparticles are taken from our experimental values $D=D_{T} / S_{T}$. The results are shown in Fig. 6 (dashed lines). Note that systematic errors exist by using $\varepsilon$ and $\eta$ of water and not including temperature and concentration dependence of $Q_{i}^{*}$.

Another theoretical equation related to the movement of a charged nanoparticle in the electrolyte gradient, in this case induced by the temperature gradient, was adapted from Prieve et al. [35]. The equation for $S_{T}^{\mathrm{NP}}(T)$ is given by

$$
S_{T}^{\mathrm{DP}}=\frac{\varepsilon k_{B} T}{D \pi \eta e}\left[\beta \zeta+\frac{4 k_{B} T}{e} \ln \left(\cosh \frac{e \zeta}{4 k_{B} T}\right)\right] S_{T}^{\text {ion }},
$$

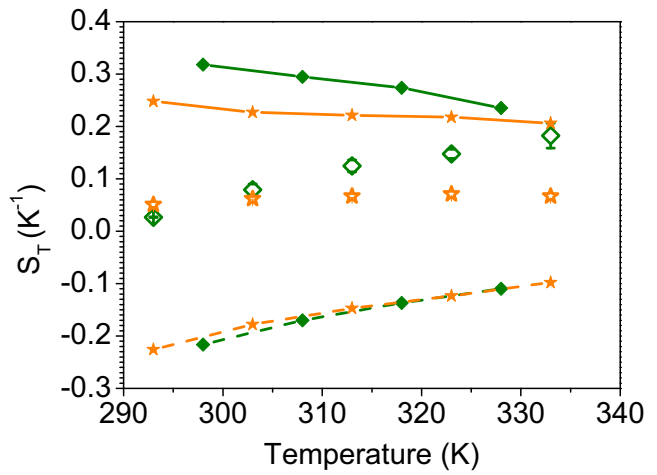

(a) where $\beta=\left(D_{+}-D_{-}\right) /\left(D_{+}+D_{-}\right), D_{+}$and $D_{-}$are, respectively, the cation and anion diffusion coefficients. Values for $D_{+}$and $D_{-}$at $T=298 \mathrm{~K}$ and ionic concentrations of $10 \mathrm{mMol} / 1$ are taken from Agar et al. [53]. As in Eq. (7) $\varepsilon$ and $\eta$ are taken from pure water, while $D$ and $S_{T}^{\text {ion }}$ are obtained from our own measurements. The $\zeta$-potential values are taken from experiments for $1 \mathrm{mM}$ of salts. For salt concentration of $20 \mathrm{mM}$ a constant value of $\zeta=-35 \mathrm{mV}$ was used for both, TMAOH and TBAOH. All the values of the physical parameters used to calculate $S_{T}^{\mathrm{NP}}$ are in the Supplemental Material [42]. The results are shown in Fig. 6 [dashed lines for Eq. (7) and solid lines for Eq. (8)]. The calculated results are shown for salt concentrations of 1 and $20 \mathrm{mM}$ due to the maximum values obtained experimentally for $S_{T}^{\mathrm{NP}}(1 \mathrm{mM})$ and to the limit of a thinner double layer $(20 \mathrm{mM})$. For salt concentrations much lower than $1 \mathrm{mM}(0.1 \mathrm{mM})$ the influence of salt gradients is still not evident, and the measured $S_{T}^{\mathrm{NP}}$ values are close to those of the original sample (see Fig. 4). The values measured for the case of $0 \mathrm{mM}$ of added salts may be related to double layer effects $[30,31]$ and are not of concern at this point to demonstrate the effect induced by salt gradients observed for high salt concentration (above

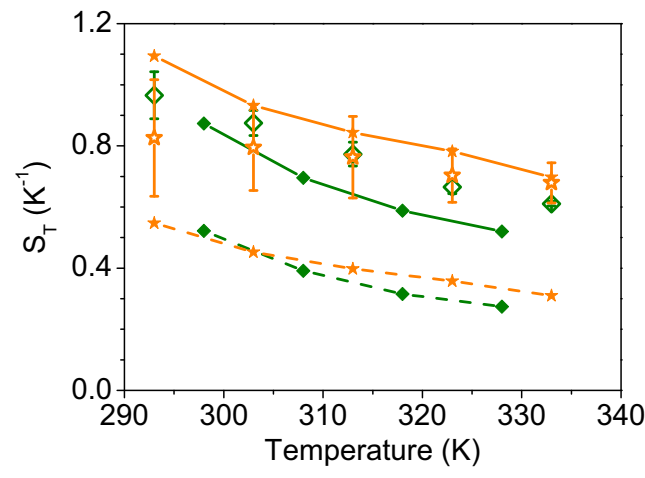

(b)

FIG. 6. Soret coefficient of the nanoparticles $S_{T}^{\mathrm{NP}}$ against temperature for $1 \mathrm{mM}$ (green diamonds) and $20 \mathrm{mM}$ (yellow stars) of TMAOH (a) and TBAOH (b). Comparison between measured values (empty symbols) and theory (small, filled symbols) according to Eq. (7) (dashed lines) and Eq. (8) (solid lines). While the theories predict the order of magnitude correctly and Eq. (8) gives satisfying agreement at high temperatures, the predicted temperature and concentration dependences of $S_{T}$ deviate strongly from the experimental data. 

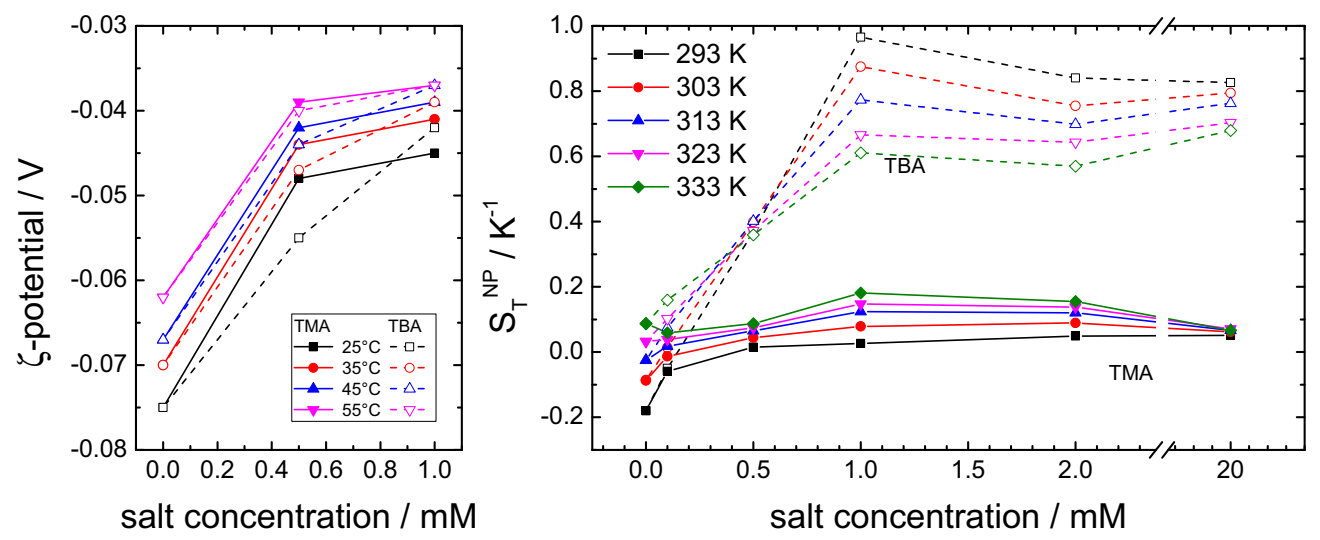

FIG. 7. Electrokinetic potential $\zeta$ and Soret coefficient $S_{T}^{\mathrm{NP}}$ of the nanoparticles against salt concentration for addition of TMAOH and TBAOH. Both parameters are sensitive to changes of NP's surface with rising salt concentrations and indicate saturation at $\approx 0.5 \mathrm{mM}$ for TMAOH and $\approx 1.0 \mathrm{mM}$ for TBAOH.

$1 \mathrm{mM}$ ). Equation (8) predicts $S_{T}^{\mathrm{NP}}$ values with the same sign and closer to experimental values for TMAOH and TBAOH, if compared with values from Eq. (7). This indicates a strong influence of the ionic Soret effect on the NP thermodiffusion, better accounted by Eq. (8). In the following, we discuss how changes in particles' surface, due to salts addition, also influences the thermodiffusive response of the charged NPs.

As discussed in the Experimental section, the ionic thermodiffusion is faster compared to that of nanoparticles, creating the electrolyte concentration gradient before the migration of nanoparticles sets in. There are several effects on the nanoparticle that occur due to the addition of the organic salts: (i) The concentration gradient of the electrolytes induces an electric field, in which the charged nanoparticle moves. (ii) The concentration gradient of the electrolyte induces a gradient in the chemical potential, which can influence the nanoparticle. (iii) The organic cations can stick to particle's surface, decrease its surface charge and hydrophilicity, and strongly modify the interaction with the surrounding solvent.

While the first two items in this list are described in Eqs. (7) and (8) through $S, \beta$, and $S_{T}^{\text {ion }}$, the third one is only partly accounted for. The change in electrokinetic potential is considered through concentration and temperature dependent experimental values of $\zeta$, but the modification of the particle's surface by the hydrophobic cations is considered in neither theory. The effect is expected to be small for inorganic ions, but organic ions like those investigated in this work can have influence due to their hydrophobicity. Previous experiments indicate that solute-solvent interactions can have a considerable contribution to the thermodiffusion behavior in water due to the existence of hydrogen bonds. We found that a decrease in hydrophilicity usually results in an increase of the Soret coefficient and a reduced or even negative temperature dependence of $S_{T}$ [54,55]. At low salt concentrations the dependence of $S_{T}^{\mathrm{NP}}$ on electrolyte concentration shown by our measurements results is inverse to what would be expected. Due to their high mobility and their thermophobic nature [29] the addition of hydroxide ions $\left(\mathrm{OH}^{-}\right)$is expected to set up a concentration gradient that drives the negatively charged NPs toward the warm side or make them less thermophobic. What we observe, however, are rising $S_{T}^{\mathrm{NP}}$ values with increasing salt concentration. This trend seems to reverse at higher salt concentrations and temperatures towards the expected behavior (cf. Fig. 7).

Trying to estimate at what concentration a full coverage (one layer) of the NP's surface by the organic cations is possible, we arrive at 0.13 and $0.61 \mathrm{mM}$ for TBA and TMA, respectively. This is, however, assuming that all cations adhere to the NP's surface. In reality, there is an equilibrium between the ions adhering to the surface and those in solution, but the equilibrium constant is not known. Indeed, due to the voluminous butyl groups surrounding the positively charged nitrogen atom, the interaction of TBA with negative charge is strongly weakened and the affinity to the NPs is likely much weaker than for TMA. This would shift the equilibrium away from the NP's surface into the solution and saturation effects due to full coverage of the surface would be expected at much higher concentrations. Figure 7 shows the electrokinetic potential $\zeta$ and Soret coefficient of the nanoparticles $S_{T}^{\mathrm{NP}}$ as a function of salt concentration for different temperatures. For both TMA and TBA we see that $S_{T}^{\mathrm{NP}}$ shows a stronger increase with concentration in the beginning, which flattens out at higher concentrations. The effect is much weaker for TMA than it is for the more hydrophobic TBA. At higher temperatures and concentrations we see a gentle decrease of $S_{T}^{\mathrm{NP}}$ with rising concentration, which might indicate that the NP's surface is saturated at that point so that its modifications stops and further concentration dependence goes in the direction predicted by Eq. (8). For the TMA we see that $S_{T}^{\mathrm{NP}}$ reaches a constant value at around $0.5 \mathrm{mM}$, which corresponds surprisingly well with the estimation of full coverage of the particles. At high temperatures, the equilibrium will be shifted towards the cations in solution, meaning a reduced adherence to the NP or a shift towards higher concentrations for the same coverage. At the same time the hydrophobic effect is less strong when hydrogen bonds are weaker, so that we expect the effect on $S_{T}^{\mathrm{NP}}$ to be less pronounced. For TBA $S_{T}^{\mathrm{NP}}$ becomes constant around $1 \mathrm{mM}$, which can also be explained by a weaker adherence of the TBA compared to the TMA. The electrokinetic potential, which is weakened (smaller absolute value) when the cations adhere to the negative surface and screen the charge, reflects the trend of the Soret coefficient measurements: at $0.5 \mathrm{mM}$ added TMA the $\zeta$ potential of the NP has almost reached a constant value, while with TBA there 
is still a marked decrease of the potential between 0.5 and $1 \mathrm{mM}$. Here too, the difference between the added salts is much weaker at higher temperatures. Note also that a $\zeta$ potential close to zero indicates weaker repulsive forces between the nanoparticles, so that aggregation becomes possible. In this low concentration range, addition of salt seems to destabilize the suspension. Assuming that a lower coverage with the organic cations results in a less hydrophobic surface of the NP and that increased hydrophobicity leads to a rise of $S_{T}^{\mathrm{NP}}$, this model can qualitatively explain the thermophoretic behavior of the NP at lower electrolyte concentrations.

The electric double layer around the charged nanoparticle also contributes to the NP's Soret effect due to the temperature dependence of the electrostatic energy stored in the double layer $[30,31,56]$. In positively charged iron oxide nanoparticles, this effect showed significant contribution when the $\zeta$ potential presents a strong temperature dependence [16]. For the samples investigated in this work the increased ionic concentration of TMAOH and TBAOH leads to very different experimental values of the $S_{T}^{\mathrm{NP}}(T)$, so that the influence of the double layer effect is not clear.

\section{v. CONCLUSIONS}

In this work we present the Soret coefficient $S_{\mathrm{T}}$ of iron oxide nanoparticles dispersed in aqueous solutions of the organic salts TMAOH and TBAOH, measured by IR-TDFRS experiments in dependence of temperature and salt concentration. A special focus lies on the influence the dispersive salts have on the thermophoresis of the nanoparticles. The typical temperature dependence of $S_{\mathrm{T}}$ described by Iacopini's empirical equation was observed for the nanoparticle in TMAOH-solution, but the equation does not hold when TBAOH is used as a dispersive salt. We show that at high concentrations thermophoresis is determined by the strong diffusiophoretic effect, which is dominant for dispersion with TBAOH. The Soret coefficient of the nanoparticles $S_{\mathrm{T}}^{\mathrm{NP}}$ can then be calculated with the ionic Soret coefficient $S_{\mathrm{T}}^{\text {ion }}$ for the salt solutions without nanoparticles, which was measured in separate experiments [47]. At low concentrations there is also an effect that is probably connected to the modification of the nanoparticles' surface by the organic cations and has not yet been described by theory. In contrast to our conclusions, some works have reported low [23] or even negligible [25] contributions from diffusiophoresis of charged particles stabilized by small monoatomic ions. The small amplitude of the Soret coefficient of these smaller ions $\left(S_{T}^{\text {ion }}\right.$ in the order of $1 \times 10^{-3} \mathrm{~K}^{-1}$ ) actually induce low particle thermophoresis, so that the discrepancy with our results is explained by the ion-specific nature of diffusiophoresis. In contrast, the amplitude of the ionic Soret coefficient for the organic salts $\left(S_{T}^{\text {ion }}(T) \sim 0.015-0.02 \mathrm{~K}^{-1}\right.$ for TMAOH and $S_{T}^{\text {ion }}(T) \sim$ $0.038-0.033 \mathrm{~K}^{-1}$ for TBAOH) is among the highest values measured for salts in general [47]. Then, diffusiophoresis becomes a main mechanism driving the Soret effect of iron oxide nanoparticles investigated in this work.

\section{ACKNOWLEDGMENTS}

The authors thank Dr. Hartmut Kriegs for his help with the experiments and Dr. Jan K. G. Dhont and Dr. Annette Schmidt for fruitful discussions. The authors acknowledge the financial support from CAPES-Br (Coordenação de Aperfeiçoamento de Pessoal de Nível Superior, Grant No. 88881.133118/201601) and INCT-FCx (Instituto Nacional de Ciência e Tecnologia de Fluidos Complexos) (CNPq, Grant No. 465259/2014-6 and FAPESP, Grant No. 2014/50983-3). D.N. acknowledges support by the International Helmholtz Research School of Biophysics and Soft Matter (IHRS BioSoft).
[1] A. Würger, Rep. Prog. Phys. 73, 126601 (2010).

[2] S. Wiegand, J. Phys.: Condens. Matter 16, R357 (2004).

[3] S. Duhr and D. Braun, Proc. Natl. Acad. Sci. USA 103, 19678 (2006).

[4] A. Würger, Phys. Rev. Lett. 101, 108302 (2008).

[5] R. Golestanian, Phys. Rev. Lett. 108, 038303 (2012).

[6] S. Iacopini and R. Piazza, Europhys. Lett. 63, 247 (2003).

[7] R. Piazza, J. Phys.: Condens. Matter 16, S4195 (2004).

[8] A. Würger, Phys. Rev. Lett. 98, 138301 (2007).

[9] Y. Zhao, C. Zhao, J. He, Y. Zhou, and C. Yang, Soft Matter 9, 7726 (2013).

[10] J. Chen, H. Cong, F.-C. Loo, Z. Kang, M. Tang, H. Zhang, S.-Y. Wu, S.-K. Kong, and H.-P. Ho, Sci. Rep. 6, 35814 (2016).

[11] L. Lin, X. Peng, Z. Mao, X. Wei, C. Xie, and Y. Zheng, Lab Chip 17, 3061 (2017).

[12] D. Vigolo, R. Rusconi, H. A. Stone, and R. Piazza, Soft Matter 6, 3489 (2010).

[13] A. Hajdú, E. Tombácz, E. Illés, D. Bica, and L. Vékás, in Colloids for Nano- and Biotechnology, edited by Z. D. Hórvölgyi and É. Kiss (Springer, Berlin, 2008), pp. 29-37.

[14] L. Zha, B. Banik, and F. Alexis, Soft Matter 7, 5908 (2011).
[15] C. Scherer and A. Figueiredo, Braz. J. Phys. 35, 718 (2005).

[16] A. L. Sehnem, A. M. Figueiredo Neto, R. Aquino, A. F. C. Campos, F. A. Tourinho, and J. Depeyrot, Phys. Rev. E 92, 042311 (2015).

[17] B. T. Huang, M. Roger, M. Bonetti, T. J. Salez, C. WiertelGasquet, E. Dubois, R. C. Gomes, G. Demouchy, G. Mériguet, V. Peyre et al., J. Chem. Phys. 143, 054902 (2015).

[18] G. Meriguet, G. Demouchy, E. Dubois, R. Perzynski, and A. Bourdon, J. Nonequilib. Thermodynam. 32, 271 (2007).

[19] K. Morozov, J. Magnet. Magnetic Mater. 201, 248 (1999).

[20] A. Mezulis, M. Maiorov, and O. Petricenko, Cent. Eur. J. Phys. 10, 989 (2012).

[21] A. L. Sehnem, R. Aquino, A. F. C. Campos, F. A. Tourinho, J. Depeyrot, and A. M. Figueiredo Neto, Phys. Rev. E 89, 032308 (2014).

[22] M. Braibanti, D. Vigolo, and R. Piazza, Phys. Rev. Lett. 100, 108303 (2008).

[23] K. A. Eslahian, A. Majee, M. Maskos, and A. Wurger, Soft Matter 10, 1931 (2014).

[24] K. A. Eslahian and M. Maskos, Colloids Surfaces A: Physicochem. Eng. Aspects 413, 65 (2012). 
[25] M. Reichl, M. Herzog, A. Götz, and D. Braun, Phys. Rev. Lett. 112, 198101 (2014).

[26] S. A. Putnam and D. G. Cahill, Langmuir 21, 5317 (2005).

[27] S. Alves, G. Demouchy, A. Bee, D. Talbot, A. Bourdon, and A. M. Figueiredo Neto, Philos. Mag. 83, 2059 (2003).

[28] O. Syshchyk, D. Afanasenkau, Z. Wang, H. Kriegs, J. Buitenhuis, and S. Wiegand, Eur. Phys. J. E 39, 129 (2016).

[29] D. Vigolo, S. Buzzaccaro, and R. Piazza, Langmuir 26, 7792 (2010).

[30] J. K. Dhont, S. Wiegand, S. Duhr, and D. Braun, Langmuir 23, 1674 (2007).

[31] J. K. G. Dhont and W. J. Briels, Eur Phys. J. E Soft Matter 25, 61 (2008).

[32] E. Bringuier and A. Bourdon, Phys. Rev. E 67, 011404 (2003).

[33] R. Piazza and A. Guarino, Phys. Rev. Lett. 88, 208302 (2002).

[34] J. L. Anderson, Ann. Rev. Fluid Mech. 21, 61 (1989).

[35] D. C. Prieve, J. L. Anderson, J. P. Ebel, and M. E. Lowell, J. Fluid Mech. 148, 247 (1984).

[36] H. Brenner, Phys. Rev. E 70, 061201 (2004).

[37] A. Würger, Langmuir 25, 6696 (2009).

[38] S. A. Putnam, D. G. Cahill, and G. C. Wong, Langmuir 23, 9221 (2007).

[39] S. Iacopini, R. Rusconi, and R. Piazza, Eur Phys J E Soft Matter 19, 59 (2006).

[40] Z. Wang, H. Kriegs, and S. Wiegand, J. Phys. Chem. B 116, 7463 (2012).

[41] D. Niether, T. Kawaguchi, J. Hovancova, K. Eguchi, J. K. G. Dhont, R. Kita, and S. Wiegand, Langmuir 33, 8483 (2017).
[42] See Supplemental Material at http://link.aps.org/supplemental/ 10.1103/PhysRevE.98.062615 for details about calculations with Eqs. (7) and (8).

[43] P. Blanco, H. Kriegs, M. P. Lettinga, P. Holmqvist, and S. Wiegand, Biomacromolecules 12, 1602 (2011).

[44] S. Wiegand, H. Ning, and H. Kriegs, J. Phys. Chem. 111, 14169 (2007).

[45] R. Kita, S. Wiegand, and J. Luettmer-Strathmann, J. Chem. Phys. 121, 3874 (2004).

[46] W. Kohler and K. I. Morozov, J. Non-Equil. Thermody. 41, 151 (2016).

[47] A. L. Sehnem, D. Niether, S. Wiegand, and A. M. F. Neto, J. Phys. Chem. B 122, 4093 (2018).

[48] D. A. Mahrouqi, J. Vinogradov, and M. D. Jackson, Geophys. Res. Lett. 43, 11578 (2016).

[49] A. F. C. Campos, R. Aquino, F. A. Tourinho, F. L. O. Paula, and J. Depeyrot, Eur. Phys. J. E 36, 42 (2013).

[50] F. Tourinho, A. Campos, R. Aquino, M. Lara, G. J. Silva, and J. Depeyrot, Braz. J. Phys. 32, 501 (2002).

[51] A. Majee and A. Würger, Soft Matter 9, 2145 (2013).

[52] A. Majee and A. Würger, Phys. Rev. Lett. 108, 118301 (2012).

[53] J. N. Agar, C. Y. Mou, and J. L. Lin, J. Phys. Chem 93, 2079 (1989).

[54] D. Niether, S. D. Lecce, F. Bresme, and S. Wiegand, Phys. Chem. Chem. Phys. 20, 1012 (2018).

[55] D. Niether, H. Kriegs, J. K. G. Dhont, and S. Wiegand, J. Chem. Phys. 149, 044506 (2018).

[56] H. Ning, J. K. Dhont, and S. Wiegand, Langmuir 24, 2426 (2008). 